www.jmscr.igmpublication.org

Impact Factor 5.84

Index Copernicus Value: 71.58

ISSN (e)-2347-176x ISSN (p) 2455-0450

crossref DOI: _https://dx.doi.org/10.18535/jmscr/v6i1.139

Journal Of Medical Science And Clinical Research

IGM Publication

An Official Publication of IGM Publication

\title{
Evaluation of Hypoalbuminemia as a Predictor of Clinical Outcome in Critically Ill Children in Alexandria University Children's Hospital
}

Authors

\author{
Azza A. Moustafa ${ }^{1}$, Aminah S. Al Halawany ${ }^{2}$, Mohamed F. Rafa ${ }^{3}$ \\ Alexandria University \\ Corresponding Author
}

Mohamed F. Rafa

Email: muhammad.rafa1985@gmail.com

\begin{abstract}
Outcome prediction is an important target in intensive care unit for providing information on patient outcomes for clinicians and family of critically ill patients. Hypoalbuminemia on admission is common in critical illness in all populations and proved to be a strong predictor of clinical outcome in adults. Because it is a simple, cheap and sensitive marker, it has been used for several purposes in adult Intensive Care Units (ICU) including outcome prediction. Although hypoalbuminemia is a common finding in critically ill children, there is a lack of data that evaluate its usefulness in predicting clinical outcome. In this retrospective study, we aimed to evaluate hypoalbuminemia as a predictor of clinical outcome in 487 critically ill children admitted to a tertiary hospital. Results showed that hypoalbuminemia was present at admission in $40.66 \%$ patients that increased to $53.8 \%$ during rest of the PICU stay. Hypoalbuminemia at admission was an independent predictor of mortality. Also, we found that patients with hypoalbuminemia at admission had a significantly higher Pediatric Logistic Organ Dysfunction score (PELOD), prolonged Pediatric Intensive Care Unit (PICU) stay and higher mortality. Patients with hypoalbuminemia during admission had significantly longer length of PICU stay, more need for mechanical ventilation, worse PELOD score, higher Pediatric Index of Mortality 2 (PIM2) score and higher mortality. Thus hypoalbuminemia at admission can be helpful for risk assessment and can be used as an outcome predictor in critically ill children.
\end{abstract}

Keywords: hypoalbuminemia; serum albumin; clinical outcome; predictor; critically ill children; pediatric intensive care unit (PICU).

\section{Introduction}

Albumin is a water-soluble protein; it is distributed in the intravascular space and twothirds in the extravascular space. It constitutes more than $50 \%$ of total plasma proteins, contributing about $80 \%$ of the plasma colloid osmotic pressure and is responsible for the transport and binding of many molecules. ${ }^{1}$ This multifunctional protein is also an important extracellular non-enzymatic antioxidant, thereby protecting against oxidative stress-induced injury. ${ }^{2}$ Abnormally low serum albumin levels are frequent and early biochemical derangements in critically ill adults with a reported incidence of $30-40 \%$; the etiology of which is complex. ${ }^{3}$

Normally, albumin has a long half-life (15-19 days), but the plasma albumin can rapidly fall by $10-15 \mathrm{~g} / \mathrm{l}$ in 3 to 5 days in critically ill patients. ${ }^{4}$ 
The inflammatory process can decrease the synthesis increase of albumin and increase its breakdown. Diversion of synthetic capacity to other acute phase reactant proteins is another cause of hypoalbuminemia in critical illnesses. During critical illness, capillary permeability increases dramatically and alters albumin exchange between intravascular and extravascular compartments. ${ }^{1,5,6}$

In a comprehensive meta-analysis of 90 studies in adult patients with acute or chronic diseases, a strong dose-response relationship was found between serum albumin level and outcome. Each $1 \mathrm{~g} / \mathrm{dL}$ decrease in serum albumin level significantly raised the odds of mortality by $137 \%$,prolonged ICU and hospital stay by $28 \%$ and $71 \%$, respectively, and increased resource utilization by $66 \%{ }^{7}$

Because it is a simple, sensitive, specific, and lowcost assay, it has been routinely used for various clinical and research purposes, including ICU outcome prediction, diagnosis, therapeutic guidance, and as a marker of severity. Large number of the studies on this issue have focused on ICU adult patients generally and specific groups of patients with a variety of pathologic conditions. $^{8}$

However, there is a paucity of data on the incidence and significance of hypoalbuminemia in critically ill children admitted to intensive care unit. This study aimed to evaluate hypoalbuminemia as a predictor of clinical outcome in critically ill children in Alexandria university children's hospital, Egypt.

\section{Subjects and Methods}

This retrospective study was conducted on the medical records of all patients admitted to the PICU which is located in a tertiary teaching hospital (Alexandria University Children's Hospital at El-Shatby) from the 1st of January 2014 to the 31 st of December 2016.

We included all patients aged more than one month and less than fourteen years admitted to the PICU whose albumin level was measured within the first 24-hours of admission. Hypoalbuminemia was defined as a serum albumin level of less than $2.5 \mathrm{~g} / \mathrm{dL}$ in infants aged less than one year and a level less than $3.4 \mathrm{~g} / \mathrm{dL}$ in older children. ${ }^{9}$

Patients who were not expected to have a normal albumin level in their usual state of health were excluded. These included:

- Patients who had severe protein-energy malnutrition (PEM) (weight for height -3 standard deviations). The Anthroplus software was used for the calculations. ${ }^{10}$

- Chronic liver disease and liver cirrhosis.

- Nephrotic syndrome and nephritis.

- Presence of a chronic gastrointestinal or kidney disease affecting the growth (malabsorption syndrome, celiac disease, inflammatory bowel disease, chronic renal failure).

- Receipt of parenteral nutrition before admission.

- Second or third-degree burns.

- Patients who received blood products or albumin before measuring the albumin level.

Baseline data collected were age, sex, weight, height, length of PICU stay, Pediatric Index of Mortality 2 (PIM2), Pediatric Logistic Organ Dysfunction (PELOD) score, diagnosis categorized by primary system affected and receipt and duration of mechanical ventilation. Our primary outcomes were the fate of patients in PICU (discharged or deceased) and length of PICU stay.

Collected data analyzed using SPSS (Statistical Package for Social Science) program for statistical analysis (version 21). Comparisons were carried out between two studied independent notnormally distributed subgroups using MannWhitney U test. ${ }^{11}$ We used Chi-square test to test the association between qualitative variables. The area under the curve (AUC) of the receiver operating characteristic (ROC) was carried out using MedCalc Software version 14. The multivariate logistic model was used to estimate the probability of a binary response based on one or more predictor. The calibration was assessed by 


\section{JMSCR Vol||06||Issue||01||Page 32299-32306||January}

directly comparing the observed and customized predicted mortality across subcategories of risk ${ }^{12}$ A $p$-value $<0.05$ was considered statistically significant.

\section{Results}

Eight hundred twenty-five patients were the final number of patients included. Four hundred eightyseven patients were the final number of included patients eligible for the study and analysis as shown in figure 1. Table- I shows demographic data of the studied patients. Table- II shows disease categories. Respiratory causes were the most common cause of admission to the PICU followed by sepsis.

As regards hypoalbuminemia at admission; the patients with were significantly older than those without ( 15 vs. $7, \mathrm{p}=0.000$ ). Patients with had a significantly longer length of PICU stay (6 vs. 4)and worse PELOD score (11 vs. 2) than those without $(\mathrm{p}=0.002, \mathrm{p}=0.000)$ respectively. There were no statistically significant differences in the need and duration of mechanical ventilation and PIM2 between the two groups $(\mathrm{p}=0.953, \mathrm{p}=0.182$, $\mathrm{p}=0.458)$ respectively. The number of deceased patients with was significantly more than those without ( 72 vs. 53, p=0.000) (Table- III).

As regards hypoalbuminemia during admission; the patients with were significantly older than those without ( 15 vs. $6, \mathrm{p}=0.000)$. Patients with had longer length of PICU stay (6 vs. 4), more need for mechanical ventilation $(63.74 \%$ vs. $53.55 \%)$, worse PELOD score (11 vs. 2) and higher PIM2 (38.75\% vs. $30 \%)$ than those without $(\mathrm{p}=0.000, \quad \mathrm{p}=0.020, \quad \mathrm{p}=0.000, \quad \mathrm{p}=0.001)$ respectively. There was no significant difference in duration of mechanical ventilation between the two groups $(p=0.106)$. The number of deceased patients with was significantly more than those without (91 vs. $34, \mathrm{p}=0.000$ ) (Table- IV).

Figure 2 and 3 shows Kaplan-Meier survival analysis of patients with and without hypoalbuminemia at and during admission. Patients with hypoalbuminemia had significantly worse survival than patients without hypoalbuminemia. $($ Log rank $=6.003, \mathrm{p}=0.014$ ) (Log rank $=4.888, \mathrm{p}=0.027)$ respectively.

Figure 4 shows the diagnostic test of the accuracy of serum albumin level at admission and the fate. The cut-off value of serum albumin at admission to predict the fate was found to be $3 \mathrm{~g} / \mathrm{dL}$ (sensitivity $72 \%$, specificity $60.5 \%$ ).

Hypoalbuminemia at admission was proved to be statistically significant independent predictors of mortality $(p=0.025)($ Table- V).

Figure 1: Flow diagram of the studied cases.

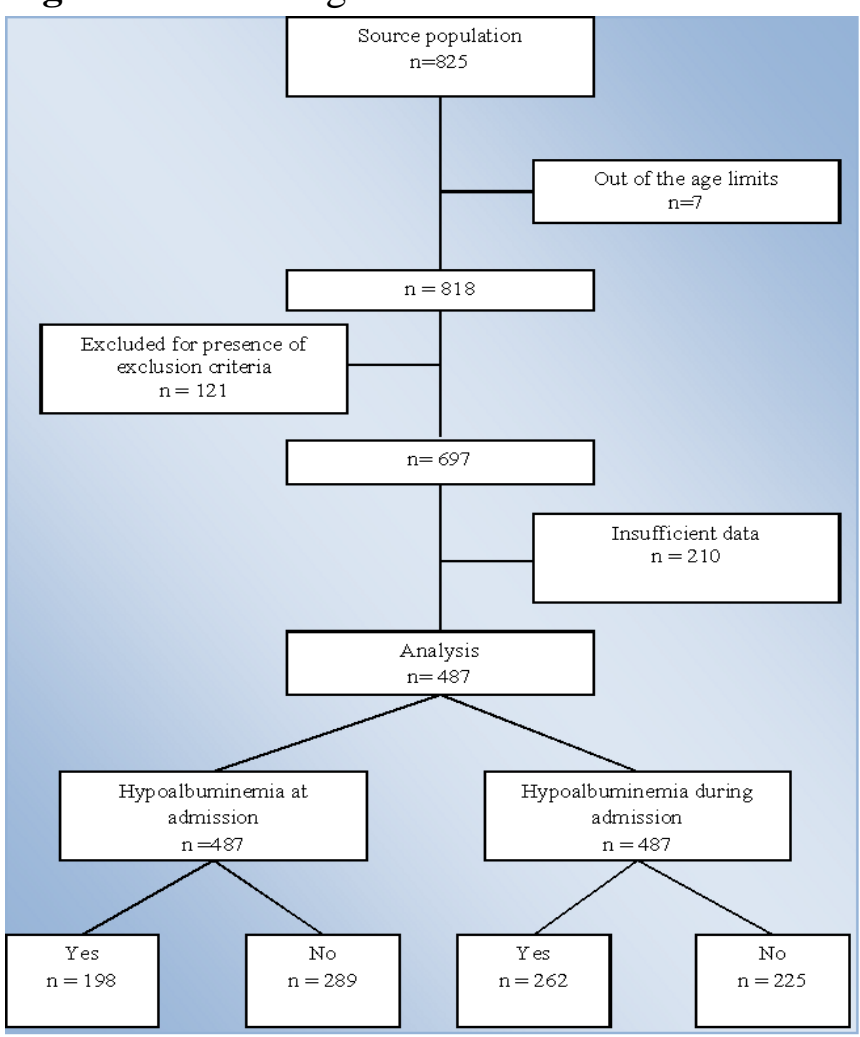

Table I: Distribution of the studied cases according todemographic data $(n=487)$

\begin{tabular}{|c|c|}
\hline & Value \\
\hline \multicolumn{2}{|l|}{ Sex } \\
\hline Male, n (\%) & $256(52.57)$ \\
\hline Age (Months) $)^{\S}$ & $9(4-28)$ \\
\hline Weight $(\mathrm{kg})^{\S}$ & $7.8(5-12)$ \\
\hline Height $(\mathrm{cm})^{\S}$ & $67(57-82)$ \\
\hline Length of PICU stay (days) $^{\S}$ & $4(2-9)$ \\
\hline Mechanical ventilation, $\mathrm{n}(\%)$ & $287(59.93)$ \\
\hline Duration of ventilation (days) ${ }^{\S}$ & $4(2-8)$ \\
\hline PELOD $^{\S}$ & $1(1-13)$ \\
\hline PIM-2 ${ }^{\S}$ & $33.50(12-64)$ \\
\hline Hypoalbuminemia at admission, $\mathrm{n}(\%)$ & $198(40.66)$ \\
\hline Hypoalbuminemia during admission, $\mathrm{n}(\%)$ & $262(53.8)$ \\
\hline Serum albumin $(\mathrm{g} / \mathrm{dL})^{\S}$ & $3.1(2.6-3.6)$ \\
\hline Mortality, n (\%) & $125(25.67)$ \\
\hline
\end{tabular}

$\S:$ Data expressed as median and interquartile range. 
Table II: Distribution of the studied cases according to primary disease $(n=487)$

\begin{tabular}{|l|c|c|}
\hline \multirow{2}{*}{ Primary disease } & \multicolumn{2}{|c|}{$(\mathrm{n}=487)$} \\
\cline { 2 - 3 } & $\mathrm{n}$ & $\%$ \\
\hline Cardiology & 36 & 7.39 \\
\hline Respiratory & 118 & 24.23 \\
\hline Neurology & 61 & 12.53 \\
\hline Renal & 14 & 2.87 \\
\hline Metabolic And Diabetes & 49 & 10.06 \\
\hline Hematology & 39 & 8.01 \\
\hline Sepsis & 94 & 19.3 \\
\hline Gastrointestinal & 55 & 11.29 \\
\hline Others & 21 & 4.31 \\
\hline
\end{tabular}

Figure 3: Kaplan-Meier survival analysis of patients with and without hypoalbuminemia during admission.

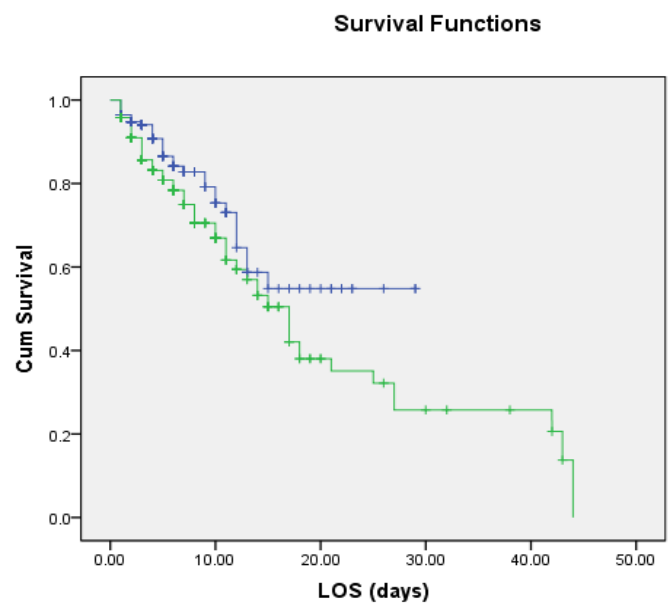

Hypoalbuminemia during admission $\neg$ NNo

+ No-censored + Yes-censored

Figure 2: Kaplan-Meier survival analysis of patients with and without hypoalbuminemia at admission.

\section{Survival Functions}
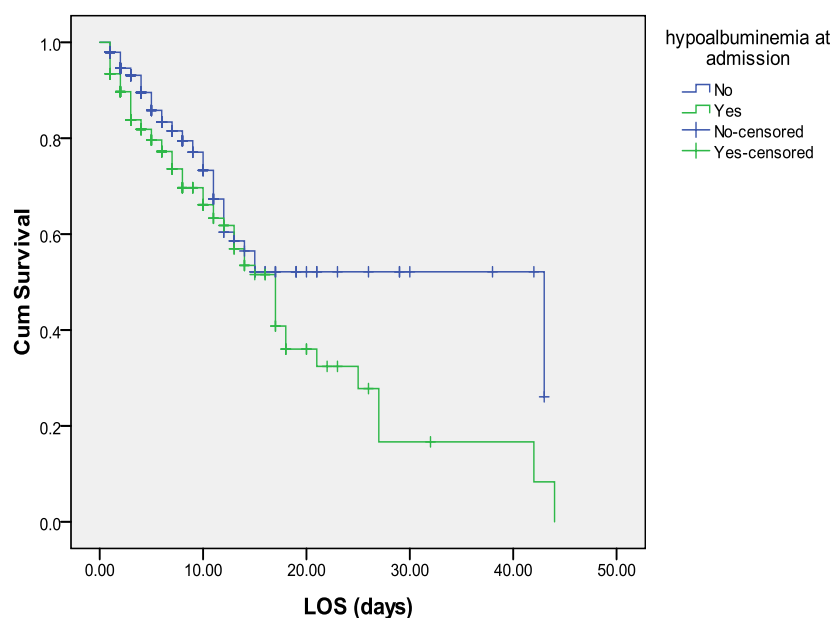

Table III: Demographic and clinical data of patients with and without hypoalbuminemia at admission.

\begin{tabular}{|c|c|c|c|c|c|c|}
\hline & \multicolumn{2}{|c|}{$\begin{array}{c}\text { Non hypoalbuminemia } \\
(\mathrm{n}=289)\end{array}$} & \multicolumn{2}{|c|}{$\begin{array}{l}\text { Hypoalbuminemia } \\
(\mathrm{n}=198)\end{array}$} & \multirow{2}{*}{$\begin{array}{c}\text { Test } \\
\text { of significant }\end{array}$} & \multirow[t]{2}{*}{$\mathrm{p}$} \\
\hline & No. & $\%$ & No. & $\%$ & & \\
\hline Male & 158 & 54.67 & 98 & 49.49 & $X^{2}=1.263$ & 0.261 \\
\hline Age (Months) ${ }^{\S}$ & \multicolumn{2}{|c|}{$7(3-14)$} & \multicolumn{2}{|c|}{$15(6-48)$} & $Z_{(\mathrm{MW}}=6.52$ & 0.000 \\
\hline Weight $(\mathrm{kg})^{8}$ & \multicolumn{2}{|c|}{$7(4.8-10)$} & \multicolumn{2}{|c|}{$9.4(6.5-15)$} & $\mathrm{Z}_{(\mathrm{MW})}=5.594$ & 0.000 \\
\hline Height $(\mathrm{cm})^{\S}$ & \multicolumn{2}{|c|}{$63(55-75)$} & \multicolumn{2}{|c|}{$74(62-93)$} & $\mathrm{Z}_{(\mathrm{MW})}=5.685$ & 0.000 \\
\hline Length of PICU stay (day) & \multicolumn{2}{|c|}{$4(2-8)$} & \multicolumn{2}{|c|}{$6(3-10)$} & $\mathrm{Z}_{(\mathrm{MW})}=3.143$ & 0.002 \\
\hline $\begin{array}{l}\text { Mechanical ventilation } \mathrm{n}(\%) \\
\mathrm{n}=287\end{array}$ & \multicolumn{2}{|c|}{$170(58.82)$} & \multicolumn{2}{|c|}{$117(59.09)$} & $X^{2}=0.003$ & 0.953 \\
\hline $\begin{array}{l}\text { Duration of mechanical } \\
\text { Ventilation (day) })^{\S}\end{array}$ & \multicolumn{2}{|c|}{$4(2-8)$} & \multicolumn{2}{|c|}{$5(2-11)$} & $\mathrm{Z}_{(\mathrm{MW})}=1.335$ & 0.182 \\
\hline PELOD $^{\S}$ & \multicolumn{2}{|c|}{$2(0-12)$} & \multicolumn{2}{|c|}{$11(1-21)$} & $\mathrm{Z}_{(\mathrm{MW})}=4.221$ & 0.000 \\
\hline PIM- $2^{\S}$ & \multirow{2}{*}{\multicolumn{2}{|c|}{$32.5(12.8-61.1)$}} & & $\mathrm{Z}_{(\mathrm{MW})}=0.742$ & 0.458 \\
\hline Mortality n(\%) & & & \multicolumn{2}{|c|}{$\frac{34.45(11.1-68.3)}{72(36.36)}$} & $X^{2}=20.008$ & 0.000 \\
\hline
\end{tabular}

$\chi 2, p: \chi^{2}$ and $\mathrm{p}$ values for Chi-square test for comparing between the two groups, $\mathrm{Z}, \mathrm{p}: \mathrm{Z}$ and $\mathrm{p}$ values for Mann Whitney test for comparing between the two groups

*: Statistically significant at $\mathrm{p}<0.05, \S$ : Data expressed as median and interquartile range. 
Table IV: Demographic and clinical data of patients with and without hypoalbuminemia during admission

\begin{tabular}{|c|c|c|c|c|c|c|}
\hline & \multicolumn{2}{|c|}{$\begin{array}{c}\text { Non hypoalbuminemia } \\
(\mathrm{n}=225)\end{array}$} & \multicolumn{2}{|c|}{$\begin{array}{l}\text { Hypoalbuminemia } \\
(\mathrm{n}=262)\end{array}$} & \multirow{2}{*}{$\begin{array}{c}\text { Test } \\
\text { of significant }\end{array}$} & \multirow{2}{*}{$\mathrm{p}$} \\
\hline & No. & $\%$ & No. & $\%$ & & \\
\hline Male & 118 & 52.44 & 138 & 52.67 & $X^{2}=0.003$ & 0.96 \\
\hline Age (Months) $)^{\S}$ & \multicolumn{2}{|c|}{$6(3-10)$} & \multicolumn{2}{|c|}{$15(6-42)$} & $Z_{(\mathrm{MW})}=7.742$ & $0.000^{*}$ \\
\hline Weight $(\mathrm{kg})^{\S}$ & \multicolumn{2}{|c|}{$6.5(4.5-9)$} & \multicolumn{2}{|c|}{$9.25(6.5-14)$} & $Z_{(M W)}=6.733$ & $0.000^{*}$ \\
\hline Height $(\mathrm{cm})^{\S}$ & \multicolumn{2}{|c|}{$61(53-70)$} & \multicolumn{2}{|c|}{$73(61-93)$} & $\mathrm{Z}_{(\mathrm{MW})}=6.79$ & $0.000^{*}$ \\
\hline Length of PICU stay (day) ${ }^{\S}$ & \multicolumn{2}{|c|}{$4(2-7)$} & \multicolumn{2}{|c|}{$6(3-10)$} & $Z_{(\mathrm{MW})}=6.733$ & $0.000^{*}$ \\
\hline $\begin{array}{l}\text { Mechanical ventilation } \mathrm{n}(\%) \\
\mathrm{n}=287\end{array}$ & \multicolumn{2}{|c|}{$120(53.33)$} & \multicolumn{2}{|c|}{$167(63.74)$} & $\mathrm{X}^{2}=5.417$ & $0.02 *$ \\
\hline $\begin{array}{l}\text { Duration of mechanical } \\
\text { Ventilation (day) }^{\S} \\
\end{array}$ & \multicolumn{2}{|c|}{$4(2-7)$} & \multicolumn{2}{|c|}{$4(2-10)$} & $\mathrm{Z}_{(\mathrm{MW})}=1.615$ & 0.106 \\
\hline PELOD $^{\S}$ & \multicolumn{2}{|c|}{$2(0-12)$} & \multicolumn{2}{|c|}{$11(2-21)$} & $\mathrm{Z}_{(\mathrm{MW})}=6.008$ & $0.000^{*}$ \\
\hline PIM- $2^{\S}$ & \multicolumn{2}{|c|}{$30(9.7-53.1)$} & \multicolumn{2}{|c|}{$38.75(17.5-69.4)$} & $Z_{(\mathrm{MW})}=3.363$ & $0.001^{*}$ \\
\hline Mortality n(\%) & \multicolumn{2}{|c|}{$34(15.11)$} & \multicolumn{2}{|c|}{$91(34.73)$} & $\square^{2}=24.427^{*}$ & $0.000^{*}$ \\
\hline
\end{tabular}

$\chi 2$, $\mathrm{p}: \chi^{2}$ and $\mathrm{p}$ values for Chi-square test for comparing between the two groups, $\mathrm{Z}, \mathrm{p}: \mathrm{Z}$ and $\mathrm{p}$ values for Mann Whitney test for comparing between the two groups

*: Statistically significant at $\mathrm{p}<0.05, \S$ : Data expressed as median and interquartile range.

Table V: Multivariate logistic regression analysis for death

\begin{tabular}{|l|c|c|c|c|}
\hline & $\mathrm{B}$ & $\mathrm{OR}$ & $95 \% \mathrm{CI}$ & $p$ value \\
\hline Age & 0.017 & 0.009 & $1.094-3.734$ & $0.025^{*}$ \\
\hline Weight & -0.07 & 0.042 & $0.859-1.012$ & 0.093 \\
\hline Hypoalbuminemia at admission & 0.703 & 0.313 & $1.094-3.734$ & $0.025^{*}$ \\
\hline Hypoalbuminemia during admission & 0.345 & 0.329 & $0.741-2.693$ & 0.294 \\
\hline PIM2 & 0.016 & 0.005 & $1.006-1.026$ & $0.002^{*}$ \\
\hline Need for mechanical ventilation & 1.658 & 0.394 & $2.425-11.357$ & $0.000^{*}$ \\
\hline PELOD & 0.062 & 0.013 & $1.038-1.092$ & $0.000^{*}$ \\
\hline Length of PICU stay & -0.019 & 0.018 & $0.947-1.017$ & 0.291 \\
\hline $\begin{array}{l}\text { Model significance } \\
\text { Classification accuracy }\end{array}$ & $\begin{array}{l} \\
X^{2}=160.837, \mathrm{p}=0.000^{*}\end{array}$ \\
\hline
\end{tabular}

B: Unstandardized Coefficients, OR: Odds ratio, CI: Confidence interval, $\chi 2, p: \chi^{2}$ and $\mathrm{p}$ values for Chi square test, *: Statistically significant at $\mathrm{p}<0.05$

Figure 4: ROC curve for serum albumin level at admission to predict deceased cases.

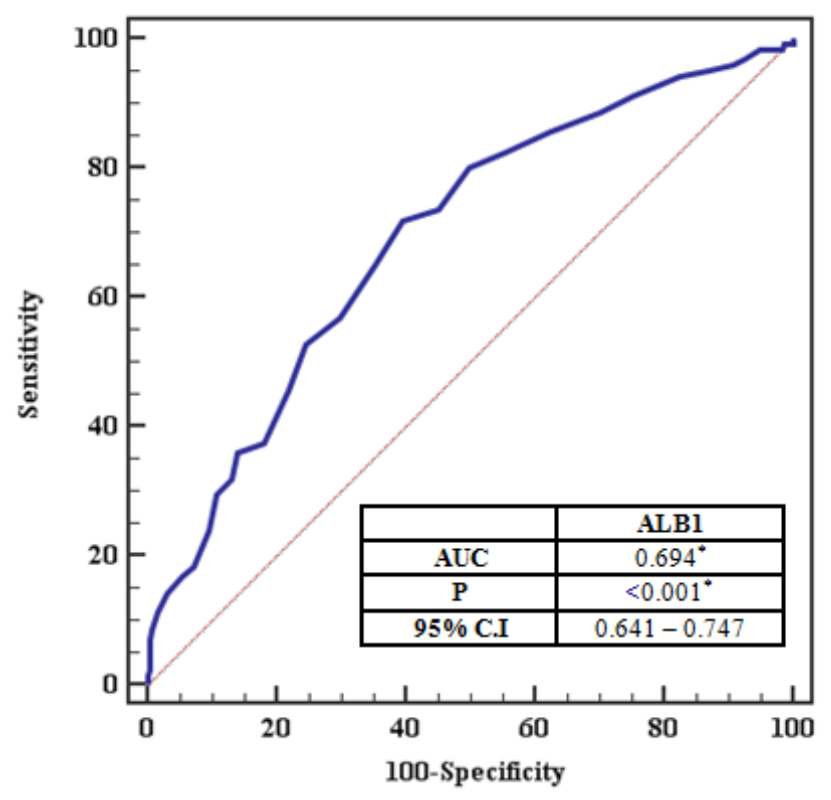

\section{Discussion}

Low albumin levels result in decreased carrier capacity for substances that are primarily transported by albumin (some medications, bilirubin, and free radicals). Because of albumin's importance in maintaining plasma colloid oncotic pressure, severe hypoalbuminemia may result in extravascular fluid accumulation. ${ }^{13}$ Also low serum albumin level is predictive of anergy, with a resultant higher incidence of infection and mortality. This is attributed to the decreased antiinflammatory and antioxidant effects of albumin. ${ }^{14}$ The incidence of hypoalbuminemia in the pediatric population is frequent. The prevalence of hypoalbuminemia in critically ill children in the previous studies ranged from $21-76 \%$. $1,3,5,6,15,16$ This might be contributed to the differences in the study population and the definition of hypoalbuminemia. 
In the current study, hypoalbuminemia was defined as a serum albumin level of less than 2.5 $\mathrm{g} / \mathrm{dL}$ in infants less than one year and a level less than $3.4 \mathrm{~g} / \mathrm{dL}$ in older children. Different definitions of hypoalbuminemia were used in the previous studies. Horowitz and $\mathrm{Tai}^{5}$, Kittisakmontri et al. ${ }^{6}$, and Kumar et al. ${ }^{16}$ defined hypoalbuminemia as a serum albumin level of less than $2.5 \mathrm{~g} / \mathrm{dL}$ in infants less than 7 months and a level less than $3.4 \mathrm{~g} / \mathrm{dL}$ in older children. Durward et al. ${ }^{3}$ defined it as lower than $3.3 \mathrm{~g} / \mathrm{dL}$. Pons Leite et al. ${ }^{15}$ defined it as less than $3.5 \mathrm{~g} / \mathrm{dL}$ while Tiwari et al. 1 defined it as less than $2.5 \mathrm{~g} / \mathrm{dL}$.

Also, there were differences in the timing of measurement that had been studied. Some of the authors used the measurement in the first 24 hours of admission in their analysis, while others used serum albumin within the first 48 hours of admission. Tiwari et al. ${ }^{1}$ studied hypoalbuminemia in the first 48 hours of admission and at any time during PICU stay. In the present study, hypoalbuminemia was analyzed within 24 hours of admission and again at any time of PICU stay. Due to these differences, a clear definition of hypoalbuminemia according to age should be made.

It is expected to see that hypoalbuminemia prevalence increases as the time of PICU stay increases as it reflects the progression and severity of the state of critical illness. In the current study, the incidence of hypoalbuminemia at admission was $40.66 \%$. This increased to $53.8 \%$ during the rest of PICU stay. Durward et al. ${ }^{3}$ reported that incidence of hypoalbuminemia increased from $57 \%$ on admission to $76 \%$ at 24 hours while Tiwari et al. ${ }^{1}$ found it increased from $21 \%$ on admission to $34 \%$ at the end of the first week and to $37 \%$ during the rest of PICU.

In this study; $36.36 \%$ of patients with hypoalbuminemia at admission died which was consistent with that of Kumar et al. ${ }^{22}$ as they reported mortality of $32 \%$. This was higher than the data from previous studies of Tiwari et al. ${ }^{1}$ and Kattisakmontri et al. ${ }^{6}$ as they reported that $25.6 \%$ to $27.5 \%$ of their patients of hypoalbuminemia at admission died. On the other hand; Durward et al. ${ }^{3}$ reported that only $12.5 \%$ of their patients with hypoalbuminemia at admission died. Regarding hypoalbuminemia during admission, the present study showed that $34.73 \%$ of them died. Tiwari et al. ${ }^{1}$ reported a lower percentage of death in the patients with hypoalbuminemia at any time of PICU stay; 23.9\%. This could be explained by the differences in the patient profile.

In the current study; patients with hypoalbuminemia at and during admission had significantly worse survival than patients without which was consistent with Horowitz and $\mathrm{Tai}^{5}$, Kattisakmontri et al. ${ }^{6}$, Pons et al. ${ }^{15}$ and Kumar et al. ${ }^{16}$ as they proved that patients with hypoalbuminemia had significantly lower survival rate. Two studies found no statistically significant difference in mortality rate between patients with hypoalbuminemia and those with normal serum albumin level. ${ }^{1,3}$ This might be attributed to the difference in definitions of hypoalbuminemia used in different studies

In adults, extensive investigations into the efficacy of hypoalbuminemia as a predictor of critical illness have been demonstrated a clear association with clinical outcomes including mortality. In the APACHE III, IV scores, albumin was added as one of the important variables in predicting prognosis. $^{17}$

Regarding mortality prediction, by using multivariate analysis, the present study found that hypoalbuminemia at admission was an independent risk factor for death. Similarly, Kattisakmontri et al. ${ }^{6}$ Pons et al. ${ }^{15}$ and Kumar et al. ${ }^{16}$ reported the same results. Also in one large cohort study where serum albumin was one of the covariates in the regression analysis, hypoalbuminemia was a significant predictor of mortality in critically ill children. ${ }^{18}$

The present study also showed that serum albumin level at admission was an important discriminator of mortality with an AUC of 0.694. In contrast; Durward et al. ${ }^{3}$ reported that the discriminatory ability of serum albumin level at admission was poor for detecting PICU mortality. 
Comparing the length of PICU stay, the current study found that PICU stay was longer in patients with hypoalbuminemia at admission and during rest of PICU stay. Similarly, many previous studies reported that patients with hypoalbuminemia at admission had a longer length of PICU stay. ${ }^{1,3,5,6,16}$

As regards to severity and progression to multiorgan dysfunction syndrome, the present study found that patients with hypoalbuminemia at admission had a significantly higher PELOD score. Similarly; Tiwari et al. ${ }^{1}$ reported that hypoalbuminemic patients had higher PRISM scores and higher likelihood progression to multiorgan dysfunction syndrome. Also, Horowitz and $\mathrm{Tai}^{5}$ found that they had a higher number of organ failures.

In contrast to some other studies; ${ }^{1,5,6,15,16}$ the current study was unable to document the difference in the patients regarding the number of ventilated cases, length of ventilation, and PIM-2 as they were similar between patients with and without hypoalbuminemia at admission.

In the present study, it was found that patients with hypoalbuminemia at any time during admission had a longer length of PICU stay, higher PIM-2, worse PELOD and more likelihood to need mechanical ventilation, but they showed no difference in duration of mechanical ventilation. Similarly; Tiwari et al. ${ }^{1}$ studied hypoalbuminemia during PICU admission. They found that patients with hypoalbuminemia had higher Pediatric Risk of Mortality scores, prolonged PICU stay; higher requiring mechanical ventilation, prolonged duration of mechanical ventilation and progression to multi-organ dysfunction syndrome.

The two limitations of the present study were: First, this study was a single center study; multicenter studies are needed to be done. Second, being a retrospective study, 210 patients $(25.4 \%)$ of the source population did not participate in the study because of incomplete data.

\section{Conclusions}

We found that hypoalbuminemia is a frequent feature in critically ill children in PICU. We also found that hypoalbuminemia at admission demonstrated a statistically significant association with clinical outcomes; longer length of PICU stay, higher PELOD score and higher mortality. Hypoalbuminemia at admission is an independent predictor of PICU mortality and serum albumin is a good discriminator for detecting mortality.

\section{References}

1. Tiwari LK, Singhi S, Jayashree M, Baranwal AK, Bansal A. Hypoalbuminemia in critically sick children. Indian J Crit Care Med. 2014;18(9):565-569. doi:10.4103/09725229.140143.

2. Roche M, Rondeau P, Singh NR, Tarnus E, Bourdon E. The antioxidant properties of serum albumin. 2008;582:1783-1787. doi:10.1016/j.febslet.2008.04.057.

3. Durward A, Mayer A, Skellett S, et al. Hypoalbuminaemia in critically ill children: incidence, prognosis, and influence on the anion gap. Arch Dis Child. 2003;88(5):419-422. doi:10.1136/adc.88.5.419.

4. Jellinge ME, Henriksen DP, Hallas P, Brabrand M. Hypoalbuminemia is a strong predictor of 30-day all-cause mortality in acutely admitted medical patients: A prospective, observational, cohort study. PLoS One. 2014;9(8). doi:10.1371/journal.pone.0105983.

5. Horowitz IN, Tai K. Hypoalbuminemia in Critically Ill Children. Arch PediatrAdolesc Med. 2007;161(11):1048. doi:10.1001/archpedi.161.11.1048.

6. Kittisakmontri K, Reungrongrat S, LaoAraya M. Hypoalbuminaemia at admission predicts the poor outcomes in critically ill children. Anaesthesiol Intensive Ther. 2016;48(3):158-161.

doi:10.5603/AIT.a2016.0028. 
7. Vincent J-L, Dubois M-J, Navickis RJ, Wilkes MM. Hypoalbuminemia in acute illness: is there a rationale for intervention? A meta-analysis of cohort studies and controlled trials. Ann Surg. 2003;237(3):319-334.

doi:10.1097/01.SLA.0000055547.93484.8 7.

8. Aman J, van der Heijden M, van Lingen A, et al. Plasma protein levels are markers of pulmonary vascular permeability and degree of lung injury in critically ill patients with or at risk for acute lung injury/acute respiratory distress syndrome. Crit Care Med. 2011;39(1):89-97. doi:10.1097/CCM.0b013e3181feb46a.

9. Soldin SJ, Brugnara C, Wong EC. Pediatric Reference Ranges.AACC Press; 2003.

https://books.google.com.eg/books?id=85J PdKwMq3QC.

10. WHO. WHO AnthroPlus software.2015. 2015:http://www.who.int/growthref/tools/e $\mathrm{n} /$.

11. Mann HB, Whitney DR. On a Test of Whether one of Two Random Variables is Stochastically Larger than the Other. Ann Math Stat. 1947;18(1):50-60. doi:10.1214/aoms/1177730491.

12.Hosmer DW, Lemeshow S, Sturdivant RX. Applied Logistic Regression, Third Edition.; 2013. doi:10.1002/0471722146.

13.Margarson MP, Soni N. Serum albumin: touchstone or totem? Anaesthesia. 1998;53(8):789-803. doi:10.1046/j.13652044.1998.00438.x.

14.Harvey KB, Moldawer LL, Bistrian BR, Blackburn GL. Biological measures for the formulation of a hospital prognostic index. Am J ClinNutr. 1981;34(10):2013-2022.

15.Leite HP, Rodrigues da Silva AV, de Oliveira Iglesias SB, Koch Nogueira PC. Serum Albumin Is an Independent Predictor of Clinical Outcomes in Critically Ill Children. PediatrCrit Care
Med. 2016;17(2):e50-7. doi:10.1097/PCC.0000000000000596.

16. Kumar S, Aroor S, Kini GP, Mundkur S, Moideen A. Hypoalbuminemia as a marker of adverse outcome in children admitted to pediatric intensive care unit. Indian J Child Heal. 2018;5(1).

17. Zimmerman JE, Kramer AA, McNair DS, Malila FM. Acute Physiology and Chronic Health Evaluation (APACHE) IV: Hospital mortality assessment for today's critically ill patients*. Crit Care Med. 2006;34(5).

http://journals.lww.com/ccmjournal/Fullte xt/2006/05000/Acute_Physiology_and_Ch ronic_Health_Evaluation.1.aspx.

18. Bai Z, Zhu X, Li M, et al. Effectiveness of predicting in-hospital mortality in critically ill children by assessing blood lactate levels at admission.BMC Pediatr. 2014;14(1):83. doi:10.1186/1471-2431-1483. 